\title{
Polarity of Liquid Interfaces by Second Harmonic Generation Spectroscopy
}

\author{
Hongfei Wang, ${ }^{\S}$ E. Borguet, $\$$ and K. B. Eisenthal ${ }^{*}$, \\ Department of Chemistry, University of Pennsylvania, Philadelphia, Pennsylvania 19104, and \\ Department of Chemistry, University of Pittsburgh, Pittsburgh, Pennsylvania 15260
}

Received: July 11, 1996; In Final Form: September 30, $1996^{\otimes}$

\begin{abstract}
A spectroscopic method, based on the interface selectivity of second-harmonic generation, is used to obtain the polarity of liquid interfaces. In this paper the second-harmonic measurement of the spectrum of the polarity indicator molecule $N, N^{\prime}$-diethyl-p-nitroaniline (DEPNA) at the air/water interface demonstrates the method. Two different approaches are used to measure the intramolecular charge transfer (CT) absorption band position of DEPNA at the air/water interface. The DEPNA CT band blue-shifts from $429 \mathrm{~nm}$ in bulk water (polar solvent) to $359 \mathrm{~nm}$ in bulk hexane (nonpolar solvent) and $329 \mathrm{~nm}$ in the gas phase (no solvent). At the air/water interface, the charge transfer peak band maximum occurs at $373 \mathrm{~nm}$, which indicates that the polarity of the air/water interface is similar to those of the bulk solvents carbon tetrachloride and butyl ether. The DEPNA results together with the results from another solvatochromic polarity indicator molecule, ET(30), which will be reported elsewhere, show that the polarity results of the air/water interface are general.
\end{abstract}

\section{Introduction}

The solvent plays a fundamental role in chemical and physical processes in bulk solution. It is well-known, for example, that the rates of chemical reactions can be increased or decreased by orders of magnitude depending on the solvent medium in which they occur. ${ }^{1-3}$ The solvent also influences other important bulk molecular behavior. The position, shape, and intensity of fluorescence bands, as well as their dynamics, are a function of the solvent medium. ${ }^{4}$ This is illustrated dramatically by species possessing TICT (twisted intramolecular charge transfer) states characterized by dual fluorescence bands, whose relative amplitude changes with the polarity of the solvent. ${ }^{5}$ The influence of solvents on chemical equilibrium constants has been known for a long time..$^{6-8}$ The dynamics and spectroscopy of intramolecular and intermolecular electron transfer processes, of practical and fundamental interest, depend on the solvent medium. ${ }^{9}$ Given the importance of the solvent in bulk processes, as indicated by the preceding examples, it is reasonable that the properties of the solvent at liquid interfaces are important in interfacial chemical and physical processes. The experiments reported here provide interface-specific measurements of solvent polarity at liquid interfaces.

Solvent polarity is the term chemists use to describe solvent effects. ${ }^{10}$ It has been defined as the "overall solvation capability of a solvent for reactants and activated complexes as well as for molecules in the ground and excited states, excluding solute-solvent interactions such as protonation, oxidation, reduction, complexation, etc., which may lead to a chemical change of the solute". ${ }^{10}$ Solvation is a complicated process that depends on a number of different solvent/solute interactions. These include nonspecific interactions arising from electrostatic and polarization forces, as well as more specific forces such as hydrogen bonding. Solvent polarity remains a difficult parameter to describe theoretically as a consequence of the diverse range of interactions involved.

However, chemists have successfully introduced useful empirical solvent polarity scales, based on solvent-dependent

\footnotetext{
* Author to whom correspondence should be addressed.

Columbia University.

¥ University of Pittsburgh.

$\S$ University of Pennsylvania.

${ }^{\otimes}$ Abstract published in Advance ACS Abstracts, January 1, 1997.
}

molecular properties. ${ }^{11-14}$ Using solvent polarity scales, quantitative correlations of solvent polarity with molecular behavior are possible. For instance, it has been shown that the dynamics of singlet to triplet intersystem crossing in aromatic carbenes can be understood and predicted using solvent polarity scales. ${ }^{15}$ Reaction rates can be predicted using empirically established linear solvation energy relationships, based on solvent polarity. ${ }^{11,12}$

The most developed and widely used solvent polarity scales are constructed from spectroscopic observations of the solvatochromic behavior of the intramolecular charge transfer (CT) $\pi-\pi^{*}$ absorption band of indicator molecules. ${ }^{10}$ Solvatochromism is the dependence of the position of a UV/vis/nearIR absorption band on the polarity of the solvent. The CT band of indicator molecules is sensitive to the solvent environment that the indicator molecule experiences. For some molecules the peak wavelength of the CT band can shift by several hundred nanometers from water, considered the most polar solvent on many of these scales, to hexane, a prototypical nonpolar solvent.

The direction and magnitude of the solvatochromic spectral shifts depend on the nature of the solvent-solute interactions. A blue (hypsochromic) shift with increasing solvent polarity is called negative solvatochromism. A red (bathochromic) shift with increasing solvent polarity is termed positive solvatochromism. The solvent effect on spectra depends on the chromophore and the nature of the transition. A red shift (positive solvatochromism) as the solvent polarity increases suggests that the excited state is more dipolar than the ground state $\left(\mu_{\mathrm{g}}<\mu_{\mathrm{e}}\right)$ (Figure 1a), where $\mu_{\mathrm{g}}$ and $\mu_{\mathrm{e}}$ are the permanent dipole moments of the ground and excited states, respectively. A blue shift (negative solvatochromism) will be observed if the ground state is more dipolar than the excited state $\left(\mu_{\mathrm{g}}>\mu_{\mathrm{e}}\right)$ (Figure 1b). In addition, there is a general red shift of the transition due to the contribution of dispersion forces to solvation of the solute molecules, which goes as $\left(n^{2}-1\right) /\left(2 n^{2}+1\right)$. This red shift is generally much smaller than the effect induced by dipole-induced dipole or dipole-dipole interactions. ${ }^{11,16-18}$ However, solvent-induced shifts of solvatochromic molecules cannot be explained by the change of the solute molecule dipole moment alone. Due to specific solute/solvent interactions such as hydrogen bonding, more than one indicator is used to probe a wide range of solvent-sensitive processes. Hence correlations 


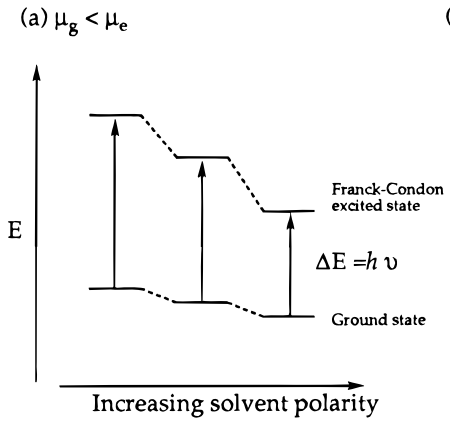

(c)<smiles>CC[N+](CC)c1ccc([N+](=O)[O-])cc1</smiles>

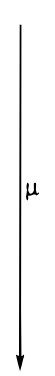

DEPNA

$\mathrm{N}, \mathrm{N}$-diethyl-p-nitroaniline (b) $\mu_{g}>\mu_{e}$

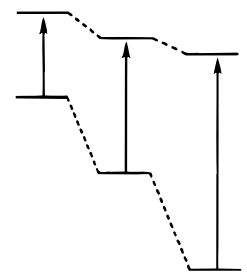

Increasing solvent polarity

(d)

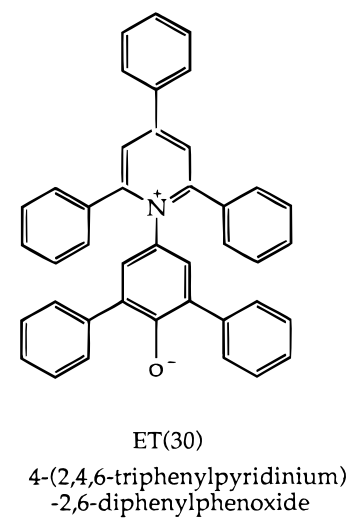

Figure 1. Qualitative representation of solvent effects on the electronic transition energy of dipolar solutes in solvents. Typical dipole moments for ground and excited states in solvent for $\mathrm{ET}(30)$ are $\mu_{\mathrm{g}}=14.7 \mathrm{D}>$ $\mu_{\mathrm{e}}=6.0 \mathrm{D}$, and for DEPNA $\mu_{\mathrm{g}}=5.1 \mathrm{D}<\mu_{\mathrm{e}}=12.9 \mathrm{D}$.

between different scales based on different indicator molecules have been established. ${ }^{11,13,19,20}$

Liquid interfaces, which are the subject of the present study, is a field of intense activity because of the scientific, environmental, and technological importance of interfaces..$^{21-24}$ The asymmetry of the forces at the interface, exemplified by the observation that molecules at liquid surfaces are aligned in a preferential direction rather than randomly oriented as they are in the bulk, ${ }^{25}$ is the origin of many of the unique chemical and physical properties of this region. ${ }^{21-24}$ Given the importance of polarity in determining chemical and physical behavior in bulk liquids, the measurement and understanding of interfacial polarity is an important scientific objective. Studies using solvatochromic polarity indicator molecules, such as ET(30) (Figure 1d), have been carried out to probe mean solvent properties of the interfacial region of water-in-oil microemulsions, ${ }^{26}$ oil-in-water microemulsions, ${ }^{27}$ and ionic and nonionic micelles, ${ }^{28,29}$ as well as alumina ${ }^{30}$ and silica ${ }^{31}$ interfaces, by UV/ vis absorption spectra measurement. ET(30) has also been investigated at free oil/water interfaces by visible attenuated total internal reflectance absorption spectroscopy. ${ }^{32}$ Unfortunately, these techniques are not necessarily surface specific. An investigation using the interface-specific technique of second harmonic generation on a well-characterized interfacial system would be helpful. This is especially true at the air/water interface, where, to our knowledge, no interface polarity measurements have been reported.

The air/water interface is a most important system and one of the most studied. It has already been demonstrated experimentally, using second-harmonic generation (SHG), that fundamental chemical behavior at interfaces, such as acid-base equilibrium constants, is different at the air/water interface from that in bulk water. ${ }^{33,34}$ The shifts of $\mathrm{p} K_{\mathrm{a}}$ showed that the air/ water interface preferentially stabilizes neutral species. ${ }^{35,36}$ Differences in solvation of ionic and neutral species at the air/ water interface undoubtedly play an important role in such equilibria. In addition, different parts of a solute molecule can experience different solvent environments at an interface. ${ }^{37}$ The discontinuity in material composition and electric field at the interface also certainly affects solvation. A knowledge of interfacial solvent polarity is important to understand and predict the properties of molecules, chemical equilibria, and dynamics of chemical reactions at interfaces.

SHG is electric dipole forbidden in centrosymmetric environments such as bulk liquids. ${ }^{21,24,38,39}$ At interfaces the inversion symmetry is broken and SHG is allowed, hence the interface specificity of SHG. The magnitude of the SHG signal, $I^{(2)}$, is proportional to the square of the second-order susceptibility, $\chi^{(2)}$, a macroscopic property of the interface (eq 1 ).

$$
I^{(2)} \propto\left|\chi^{(2)} E_{\omega} E_{\omega}\right|^{2}
$$

where $E_{\omega}$ is the amplitude of the incident field at the frequency $\omega$.

$\chi^{(2)}$ can be expressed as a sum over all the states $(\mathrm{a}, \mathrm{b}, \mathrm{c}, \ldots)$ of the molecular system that interact with the fundamental laser frequency, $\omega$, and its second harmonic, $2 \omega .{ }^{40}$ One of the most important terms in $\chi^{(2)}$ for the purposes of this work is

$$
\chi^{(2)}=\sum_{\mathrm{b}, \mathrm{c}} \frac{\mu_{\mathrm{ab}} \mu_{\mathrm{bc}} \mu_{\mathrm{ac}}}{\left(\omega_{\mathrm{ba}}-\omega+\mathrm{i} \Gamma_{\mathrm{ab}}\right)\left(\omega_{\mathrm{ca}}-2 \omega+\mathrm{i} \Gamma_{\mathrm{ac}}\right)}
$$

where $\mu_{\mathrm{ab}}$ is the transition dipole moment, $\omega_{\mathrm{ba}}$ is the optical frequency of the transition, and $\Gamma_{a b}$ is the linewidth associated with the transition.

Resonance enhancement of $\chi^{(2)}$ occurs when the fundamental laser frequency, $\omega$, and/or its second harmonic, $2 \omega$, is in resonance with a transition between molecular states. This typically increases experimental signals by several orders of magnitude. ${ }^{41}$ By tuning the incident laser light frequency, one can measure $\chi^{(2)}$ as a function of photon energy, yielding interfacial spectroscopic information. A number of investigations have exploited the spectroscopic character of $\mathrm{SHG}^{41-43}$ and visible sum frequency generation (SFG), ${ }^{4,45}$ a related surface nonlinear technique, to reveal the electronic transition spectra of interfacial species.

In this paper we present the results of experiments designed to determine the polarity of the air/water interface. This is achieved by the $\mathrm{SH}$ spectroscopy of the polarity indicator molecule, DEPNA ( $N, N$-diethyl- $p$-nitroaniline). Two different approaches are used. One approach is based on the SH spectrum obtained at a fixed surface density of DEPNA. The other approach exploits the different density dependence of the $\mathrm{SH}$ signal at different wavelengths. The use of two methods provides a self-consistent check on the accuracy of the polarity determination. It also enables us to determine if the spectra are dependent on the density of DEPNA at the air/water interface, in the density range of our experiments. We have chosen to use DEPNA because its solvent-induced absorption peak shifts are large in bulk solution and within an experimentally accessible range for the tunable laser system used in this study. In addition, DEPNA is stable in bulk water and exists predominately in its neutral form over a wide range of solution $\mathrm{pH}$. DEPNA is a positively solvatochromic species and one of the most extensively studied nitroaromatic indicators. ${ }^{19,20}$ The solvatochromic behavior of the nitroaromatic indicators is associated with a $\pi-\pi^{*}$ CT transition, involving charge density at the nitro and anilino substituents. Based on these nitroaromatic compounds, a series of correlated $\pi^{*}$ scales of solvent polarity have been constructed. For DEPNA the $\pi-\pi^{*}$ band 


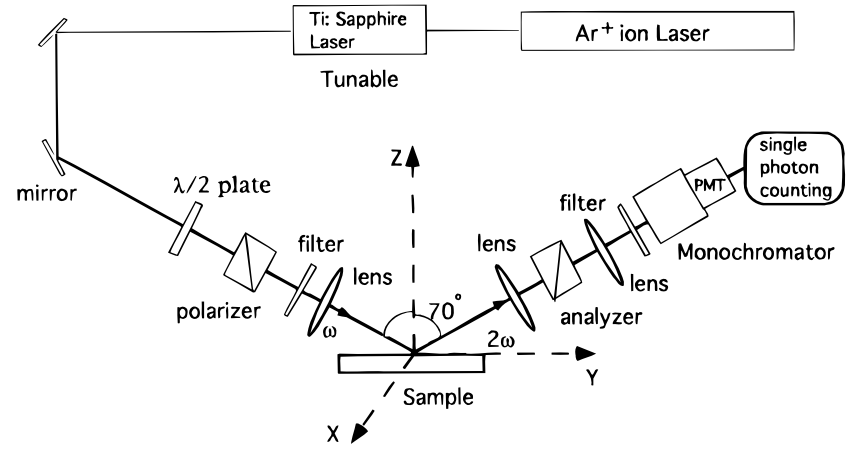

Figure 2. Experimental setup for SHG.

peaks at $429 \mathrm{~nm}$ in bulk water, the most polar solvent in the scale, and $359 \mathrm{~nm}$ in bulk $n$-hexane, the most nonpolar solvent in the scale. ${ }^{47}$

\section{Experimental Methods}

The SHG experimental setup is shown in Figure 2. A Ti: sapphire oscillator (Spectra-Physics Tsunami), pumped by an $\mathrm{Ar}^{+}$ion laser (Spectra-Physics 2080), provides $100 \mathrm{fs}$ pulses at a repetition rate of $82 \mathrm{MHz}$. The output of the Ti:sapphire laser is tunable from 720 to $900 \mathrm{~nm}$, with a bandwidth of about 10 $\mathrm{nm}$. The polarization of the incident beam is controlled using a half-wave plate and polarizer. A long-wavelength pass filter in the incoming path blocks any second-harmonic light generated from preceding optical components. The fundamental light is focused to a $10 \mu \mathrm{m}$ diameter spot at the sample surface. The typical power at the sample is about $0.6 \mathrm{~W}$. Second-harmonic photons are detected in the reflected direction using singlephoton counting. The short-wavelength pass filter and monochromator serve to separate the second harmonic from the fundamental and background (two-photon fluorescence, ...) radiation. Two distinct elements of $\chi^{(2)}, \chi^{(2)}{ }_{Z X X}$ and $\chi_{X Z X}^{(2)}$, can be independently probed using the polarizer and analyzer pair. The subscript $Z$ refers to the laboratory axis perpendicular to the interface, and $X$ and $Y$ are axes parallel to the surface, as shown in Figure 2.

The SH signal $\left(I^{(2)}\right)$ from the air/water interface of indicator solutions was normalized to the $\mathrm{SH}$ signal of the air/neat water interface, which serves as a wavelength-independent reference. The normalization for each element of the susceptibility directly yields the square of $\chi^{(2) D E P N A} / \chi^{(2) \text { Water }}$, and hence the dependence of $\chi^{(2) \text { DEPNA }}$ on wavelength as $\chi^{(2) \text { Water }}$ is constant over the wavelength region investigated. The normalized $\mathrm{SH}$ signal is noted as $I_{I J K}^{(2) \text { DEPNA }}$ in the text, where $I J K$ is $Z X X$ or $X Z X$. In addition, this normalization to the signal of a known reference interface (neat water/air) allows us to estimate the absolute population of indicator molecules at the air/water interface, as discussed later.

DEPNA was initially synthesized and purified by a standard literature procedure. ${ }^{46}$ Later it was purchased (Frinton Laboratories, $\mathrm{NJ}$ ) and used without further purification. Both samples gave the same experimental results. Solution $\mathrm{pH}$ was adjusted to between 3.5 and 4.0 using $\mathrm{HCl}$ (Amend Drug and Chemical Co.) to ensure that the neutral polarity indicator form $\left(\mathrm{p} K_{\mathrm{a}}<\right.$ 2) predominates. Double-distilled water was used to prepare all solutions. All experiments were performed at $22 \pm 0.5^{\circ} \mathrm{C}$.

\section{Results and Discussion}

The SH spectrum of DEPNA at the air/water interface, $I_{X Z X}^{(2) D E P N A}$ (filled circles) and $I_{Z X X}^{(2) D E P N A}$ (open circles), for a bulk concentration of $30 \mu \mathrm{M}$, is plotted vs the SH wavelength in Figure 3a. By inspection both $I_{X Z X}^{(2) D E P N A}$ and $I_{Z X X}^{(2) \text { DEPNA }}$ peak at

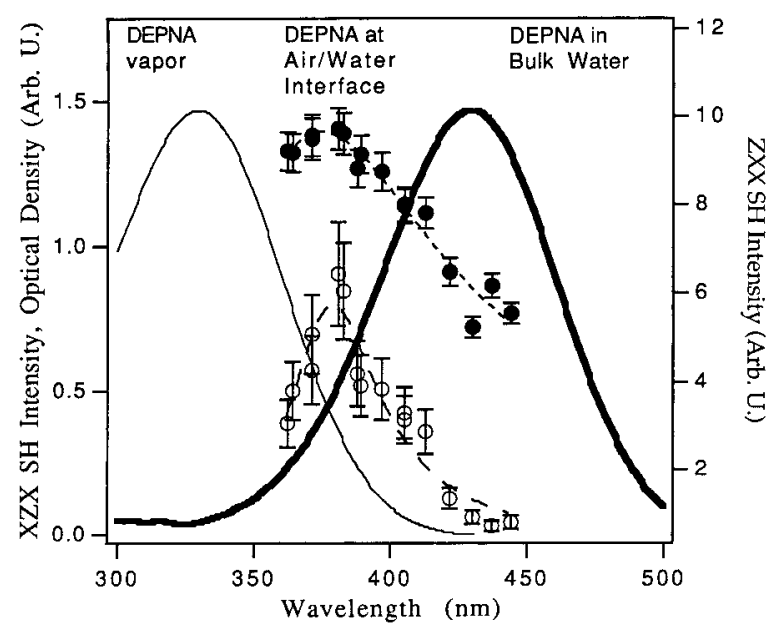

b)

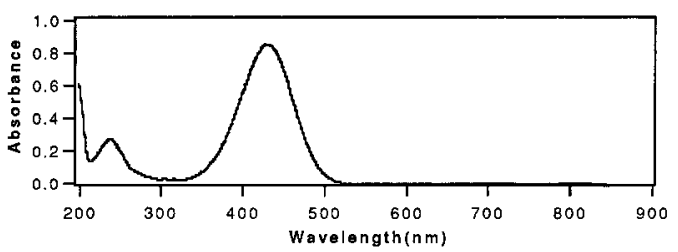

Figure 3. Normalized second-harmonic spectra $(\triangle X X$ and $\bigcirc Z X X)$ of DEPNA at the air/water interface. Bulk concentration is $30 \mu \mathrm{M}$. The absorption spectrum of DEPNA in bulk water peaks at $429 \mathrm{~nm}$, while in air it peaks at $329 \mathrm{~nm}$. Two dotted lines are fittings of $I_{X Z X}^{(2) D E P N A}$ and $I_{Z X X}^{(2) D E P N A}$ to eq 4. (b) Absorption spectrum of DEPNA in bulk water.

about $377 \mathrm{~nm}, 52 \mathrm{~nm}$ from the absorption peak of DEPNA in bulk water. The interface peak is similar to the peak position of DEPNA in media such as bulk carbon tetrachloride $(375 \mathrm{~nm})$, a nonpolar solvent. ${ }^{47}$ This suggests that the environment at air/ water interface is significantly less polar than bulk water.

As the signals from the interface of neat water and aqueous DEPNA solutions are of the same order of magnitude, the contribution of both water and DEPNA molecules must be considered in the analysis. $I_{I J K}^{(2)}$ can be written as

$$
\begin{aligned}
I_{I J K}^{(2)} & \propto \mid \chi_{I J K}^{(2) \text { Water }}+\chi_{I J K}^{(2) \text { DEPNA }\left.\right|^{2}} \\
& \propto\left|\chi_{I J K}^{(2) \text { Water }}+\chi_{I J K, \text { Resonant }}^{(2) \text { DEPNA }}+\chi_{I J K, \text { Nonresonant }}^{(2) \text { DEPNA }}\right|_{\tau}^{2}
\end{aligned}
$$

where the resonant and nonresonant parts of $\chi_{I J K}^{(2) D E P N A}$ are explicitly written. Only the resonant term $\chi_{I J K, \text { Resonant }}^{(2) D E P N A}$ is a strong function of wavelength. Because the laser used in the present study is tunable in the range $720-900 \mathrm{~nm}$, it is only the two-photon transitions between the ground and the excited states of DEPNA at twice the incident laser frequency that can yield a resonant enhancement of the $\mathrm{SH}$ signal. The most important term for $\chi^{(2) \text { DEPNA }}$ in the vicinity of a two-photon resonance involving the ground state is given by eq 2 . As DEPNA has only one absorption band in the whole visible and near-IR region, viz., Figure $3 b$, we need only consider the $2 \omega$ resonant transition term, around $400 \mathrm{~nm}$, in eq 2. Normalizing the $\mathrm{SH}$ intensity to that of the air/neat water interface yields

$$
\begin{aligned}
I_{I J K}^{(2) \mathrm{DEPNA}} & =\left|1+\frac{a}{\omega_{\max }-2 \mathrm{w}+\mathrm{i} \Gamma}+b\right|^{2} \\
& =\left|1+\frac{a^{\prime}}{\frac{1}{\lambda_{\max }}-\frac{2}{\lambda}+\mathrm{i} \Gamma^{\prime}}+b\right|^{2}
\end{aligned}
$$


TABLE 1: Spectral Parameters (Eq 4) of $\chi_{X Z X}^{(2) D E P N A}$ and $\chi_{\text {ZXX }}^{(2) \text { DEPNA }}$

\begin{tabular}{ccccc}
\hline element & $a^{\prime}$ & $b$ & $\Gamma^{\prime}$ & $\lambda_{\max }(\mathrm{nm})$ \\
\hline$\chi_{X Z X}^{(2) \text { DEPNA }}$ & 0.00046 & -0.87 & 0.00039 & $369 \pm 3.0$ \\
$\chi_{\text {ZXX }}^{(2) \text { DEPNA }}$ & 0.00035 & -0.83 & 0.00015 & $376 \pm 6.4$
\end{tabular}

where $a^{\prime}, b, \Gamma^{\prime}$, and $\lambda_{\max }$ are the resonance amplitude of $\chi_{I J K, \text { Resonant }}^{(2) \text { and }} \chi_{I J K, \text { Nonresonant }}^{(2) \text { DEPNA }}$, the linewidth, and the transition peak wavelength, respectively. $\lambda$ is the fundamental (laser) wavelength. The first term inside the absolute value brackets in eq 4 , i.e. 1 , is due to water, and the second and third terms are the resonant and nonresonant parts of $\chi_{I J K}^{(2) \text { DEPNA }}$. The results of the fittings to eq 4 for $I_{X Z X}^{(2) \text { DEPNA }}$ (dash-dotted line) and $I_{Z X X}^{(2) D E P N A}$ (dashed line) are shown in Figure 3a. The fitting parameters are summarized in Table 1.

The average transition peak wavelength, $\lambda_{\max }$, which is obtained from the fitting to eq 4 , of $\chi_{X Z X}^{(2) D E P N A}$ and $\chi_{Z X X}^{(2) D E P N A}$ is $373 \pm 4 \mathrm{~nm}$. The $\lambda_{\max }$ contained in eq 4 corresponds to the energy separation between the charge transfer and the ground states. In a linear absorption measurement $\lambda_{\max }$ is the peak in the absorption spectrum. The measured normalized SH signals, $I_{X Z X}^{(2) D E P N A}$ and $I_{Z X X}^{(2) D E P N A}$, peak about $4 \mathrm{~nm}$ to the red of the average $\lambda_{\text {max }}$. This difference between the observed peaks in the linear and $\mathrm{SH}$ spectra has previously been noted in measurements of a Langmuir-Blodgett (L-B) monolayer of 4' (n-phenyl)-4-cyano- $p$-terphenyl (T-15) on fused silica. ${ }^{45}$ The difference between the linear and the $\mathrm{SH}$ spectrum results from the interference of resonant and non resonant terms of the second-order susceptibility. This effect is absent in linear absorption. In the study of T-15 on fused silica the peak of the linear spectrum was found at $286 \mathrm{~nm}$, and the $\mathrm{SH}$ spectrum peak at $280 \mathrm{~nm} .{ }^{45}$ The $56 \mathrm{~nm}$ shift of $\lambda_{\max }$ for DEPNA at the air/water interface relative to bulk water is due to the different energy separations between the ground and lowest excited CT states of DEPNA at the interface and in the bulk. It is not a consequence of the difference between $\mathrm{SH}$ and linear absorption spectroscopy. The apparent peak of the SH spectrum is the maximum value of the $I_{I J K}^{(2) D E P N A}$, which contains contributions from both the resonant $\left(a^{\prime}\right)$ and nonresonant $(1+b)$ terms. The nonresonant part is due to $\mathrm{H}_{2} \mathrm{O}$ and DEPNA states far from resonance. The nonzero value of $b$ indicates that there is a significant nonresonant contribution term to the second-order susceptibility from the solvated complex. Inspection of eq 4 indicates that the apparent $\mathrm{SH}$ peak will be to the red of $\lambda_{\max }$ as long as $a^{\prime}$ and $(1+b)$ have the same sign. As noted earlier, $\lambda_{\max }$ determined by fitting eq 4 is identical to the peak wavelength that would be seen if the linear absorption spectrum of the interface molecules were measured. $a^{\prime}$ and $b$ are both negative, clearly indicating that $\chi^{(2) D E P N A}$ has opposite phase to that of $\chi^{(2) \text { Water }}$ at photon energies below resonance. Taking into account these effects, the surface SH spectrum reveals that the peak of the electronic CT transition for DEPNA molecules at the air/water interface is blue shifted by $56 \mathrm{~nm}$ from its position in bulk water.

An alternative means of determining the peak of the CT transition at the air/water interface is based on the dependence of the SH intensity on the density of DEPNA at the interface. The method, described below, uses a smaller number of parameters to fit the data as well as an increased data set, as it draws on the spectra at several different densities. It provides a selfconsistent check on the accuracy of our measurement. It also verifies that the peak of the $\mathrm{CT}$ transition at the air/water interface is independent of the density of DEPNA at the interface.

The surface tension of aqueous DEPNA solutions, for bulk

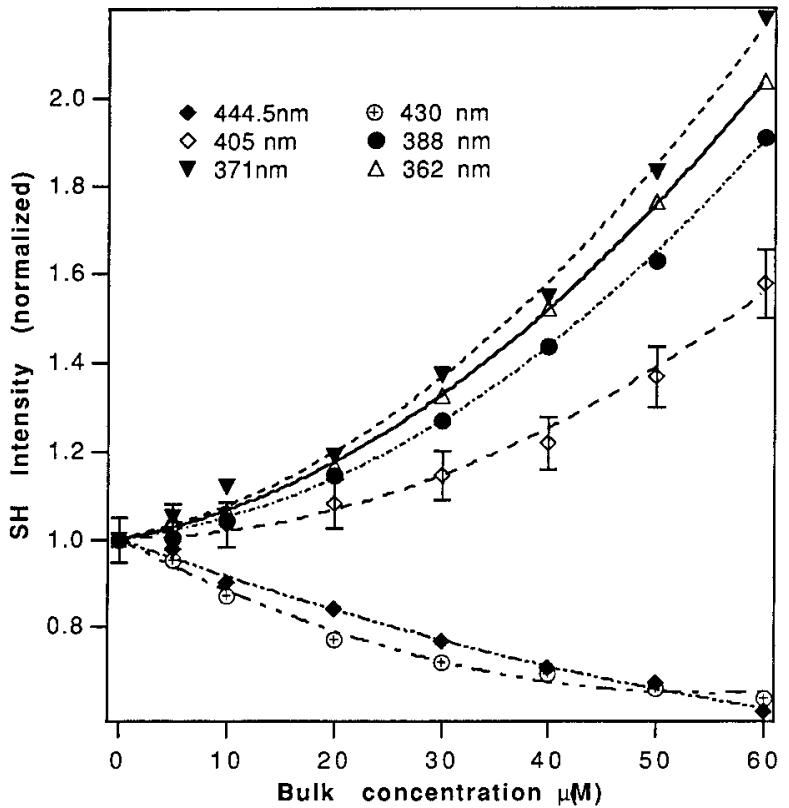

Figure 4. Normalized second-harmonic adsorption isotherm for $I_{X Z X}^{(2) D E P N A}$ at different wavelengths. The symbols are the experimental data; the lines are fittings using the linear dependence of the interface density of DEPNA on its bulk concentration for the concentration range studied. The SH intensity increases with decreasing wavelength until $371 \mathrm{~nm}$; then it decreases from 371 to $362 \mathrm{~nm}$, indicating a peak in the $\mathrm{SH}$ spectrum.

concentrations from 1 to $60 \mu \mathrm{M}$, is the same as that of neat water, within experimental accuracy $( \pm 0.1 \mathrm{dyn} / \mathrm{cm})$. This indicates a lower limit in the density of $4200 \AA^{2} /$ DEPNA molecule at $60 \mu \mathrm{M} .{ }^{48}$ The surface tension measurements are consistent with the low density of DEPNA at the air/water interface, inferred from the low SH intensity. The low $\mathrm{SH}$ intensity is not a consequence of DEPNA molecules laying flat at the interface, as the polarization of the $\mathrm{SH}$ signal shows that the principal axis of DEPNA is oriented at about $55^{\circ}$ from the surface normal. At these low densities the adsorption of DEPNA to the air/water interface is in the linear region of the adsorption isotherm; that is, the interface density of DEPNA is linearly proportional to its bulk concentration. The UV/vis absorption spectra of DEPNA in bulk water at concentrations up to $60 \mu \mathrm{M}$ obey Beer's law and show no evidence of dimerization or aggregation in the bulk. The linear relationship between the surface density and the bulk concentration, verified by the SHG isotherm, to be described below, indicates that there is no aggregation in the interface up to a bulk concentration of $60 \mu \mathrm{M}$.

In addition to being noninvasive, our results show that SHG is a more sensitive way to obtain the adsorption isotherm than surface tension measurements. ${ }^{49}$ The $\mathrm{SH}$ isotherm for $I_{X Z X}^{(2) D E P N A}$ as a function of bulk DEPNA concentration at selected wavelengths, below, at, and above resonance, is shown in Figure 4. $I_{Z X X}^{(2) D E P N A}$ showed similar behavior. The strong wavelength dependence of the data clearly shows the effect of the resonance on the $\mathrm{SH}$ adsorption isotherm. The contribution of both water and DEPNA molecules to the SH signal must be considered. To facilitate the analysis, the real and imaginary parts of the DEPNA susceptibility are separated explicitly:

$$
\begin{aligned}
I_{I J K}^{(2)} \propto\left|\chi_{I J K}^{(2) \text { Water }}+\chi_{I J K}^{(2) \text { DEPNA }}\right|^{2} \propto \mid \chi_{I J K}^{(2) W a t e r}+ & \chi_{I J K, \text { Real }}^{(2) \text { DEPNA }}+ \\
& \left.\iota \chi_{I J K, \text { Imaginary }}^{(2) \text { DEPNA }}\right|^{2}
\end{aligned}
$$

$\chi_{I J K, \text { Real }}^{(2) \text { DEPNA }}$ is the sum of the nonresonant term $\chi_{I J K, \text { Nonresonant }}^{(2) \text { DEPNA }}$ and 
TABLE 2: Wavelength Dependence of the Real $(A)$ and Imaginary $(B)$ Parts of the DEPNA Second-Order Polarizability (Eq 7)

\begin{tabular}{lrrcccc}
\hline & \multicolumn{6}{c}{ second-harmonic wavelength (nm) } \\
\cline { 2 - 7 } & \multicolumn{1}{c}{445} & \multicolumn{1}{c}{430} & 405 & 388 & 371 & 362 \\
\hline$A$ & -0.0045 & -0.0064 & 0.0000 & 0.0013 & 0.0024 & 0.0022 \\
$B$ & 0.0047 & 0.0087 & 0.0124 & 0.0143 & 0.0155 & 0.0140
\end{tabular}

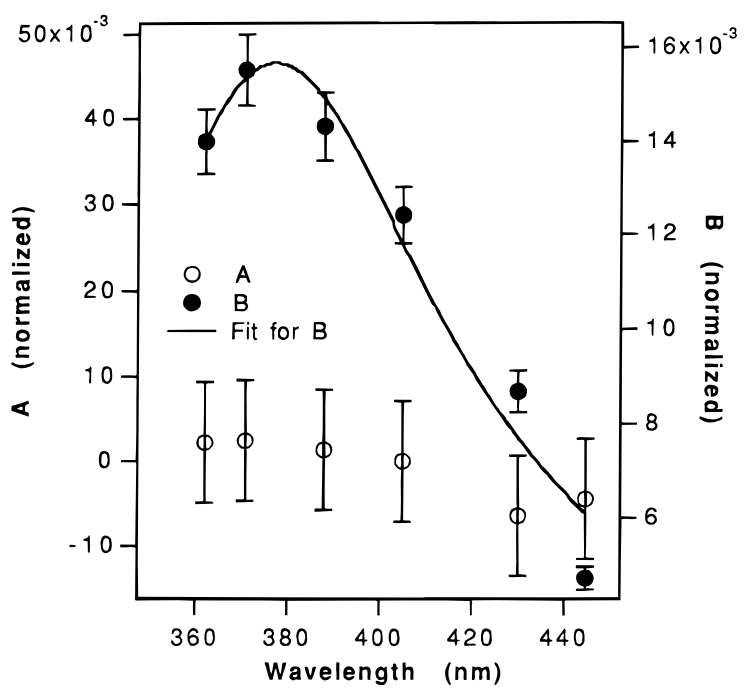

Figure 5. Plot of $A$ and $B$ from eq 7 vs wavelength. Fitting of $B$ gives a peak at $376 \pm 5 \mathrm{~nm}$, consistent with the $\mathrm{SH}$ spectrum (Figure $3 \mathrm{a}$ ).

the real part of the resonant term $\chi_{I J K, \text { Resonant }}^{(2) \text { DEPNA }}$ in eq 4. $\chi_{I J K, \text { Imaginary }}^{(2) \text { is }}$ is the imaginary part of the resonant term $\chi_{\text {(2)DEPNA }}$

Because the SHG measurements are performed in the linear region of the adsorption isotherm, the nonlinear susceptibility $\chi^{(2) D E P N A}$ is proportional to the bulk DEPNA concentration, $C$.

$$
\chi^{(2) \mathrm{DEPNA}}=N_{\text {Surface }}^{\mathrm{DEPTA}} \alpha^{(2) \mathrm{DEPNA}} \approx C\left\langle\alpha^{(2) \mathrm{DEPNA}}\right\rangle_{\text {Orientation }}
$$

where $N_{\text {Surface }}^{\text {DEPA }}$ is the density of DEPNA at the interface, and $\alpha^{(2) D E P N A}$ is the second-order susceptibility of DEPNA.

Normalization to the air/neat water interface, using eq 6 , simplifies eq 5 to

$$
I_{I J K}^{(2) \mathrm{DEPNA}}=|1+A C+\mathrm{i} B C|^{2}=(1+A C)^{2}+(B C)^{2}
$$

where $A$ and $B$ are proportional to the real and imaginary part of the DEPNA molecular second-order polarizability, $\alpha^{(2) D E P N A}$, respectively. $A$ and $B$ are wavelength dependent, but are independent of interface density. The fits shown in Figure 4 indicate that eq 7 correctly models the adsorption isotherms at each wavelength. The nonlinear, nonmonotonic behavior is a consequence of the interference between the susceptibility of water, whose phase is constant, and that of DEPNA, whose phase varies with wavelength. The values determined for $A$ and $B$ from $I_{X Z X}^{(2) D E P N A}$ are reported in Table 2 and plotted in Figure 5 as a function of measured wavelength.

$B$, the imaginary part of the DEPNA susceptibility, increases with decreasing wavelength as the resonance at $373 \mathrm{~nm}$ is approached and then turns over beyond the resonance peak position. Because at the longer wavelegths, namely, 445 and $430 \mathrm{~nm}, A$ is of opposite sign to the contribution from the water background, it follows that $|1+A C|<1$. This explains why at 445 and $430 \mathrm{~nm}$, where $|B|$ is small, the $\mathrm{SH}$ isotherm actually goes below the neat air/water value, which is unity, as the bulk concentration of DEPNA increases, Figure 4. At shorter wavelengths where $B$ is larger, $I_{X Z X}^{(2) D E P N A}$ goes above the value for the neat water/air interface. $A$ contains contributions from both the real part of the resonant term of $\chi_{I J K, \text { Resonant }}^{(2) \text { DEPNA }}$ and the

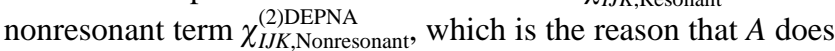
not show the pure resonant behavior that $B$ does. Since $B$ only contains contributions from the resonant terms of the susceptibility, fitting of $B$ as a function of $\mathrm{SH}$ wavelength should give the peak position of the resonance. In Figure 5 the solid line is a fit to eq 5 , yielding a peak position of $376 \pm 5 \mathrm{~nm}$. This result is consistent with the $373 \pm 4 \mathrm{~nm}$ value of $\lambda_{\max }$, obtained from the fitting to eq 4 . The agreement between the values obtained for the two different methods is good and provides a self-consistent check on the accuracy of the determination of the polarity of the air/water interface.

The interface spectrum can be related to the DEPNA solvent polarity scale. The correlated $\pi^{*}$ value for DEPNA is given by eq $8 .{ }^{19}$

$$
\pi^{*}=\frac{v_{\max }-27.52}{-3.182}
$$

$v_{\max }$ is the absorption band peak in units of $10^{3} \mathrm{~cm}^{-1}$. On this scale, bulk water has a $\pi^{*}$ value of 1.35 and $n$-hexane is -0.06 . In the gas phase DEPNA peaks at $329 \mathrm{~nm}$, corresponding to a $\pi^{*}$ value of $-0.90 .{ }^{50}$ The peak wavelength of DEPNA at the air/water interface, $373 \pm 4 \mathrm{~nm}$, yields a polarity value of $\pi^{*}$ $=0.22 \pm 0.07$ (viz., eq 8). The polarity of the air/water interface appears very different from that of bulk water $\left(\pi^{*}=\right.$ $1.35)$, a very polar medium, and that of the vapor phase $\left(\pi^{*}=\right.$ $-0.90)$. The measured polarity of the air/water interface $(0.22$ $\pm 0.07,373 \pm 4 \mathrm{~nm})$ is similar to that of nonpolar solvents such as carbon tetrachloride $\left(\pi^{*}=0.26,375 \mathrm{~nm}\right)$ and butyl ether $\left(\pi^{*}=0.21,372 \mathrm{~nm}\right){ }^{47}$

Spectral shifts at the air/water interface were also observed for ET(30), a negative solvatochromic polarity indicator molecule. The polarity of the air/water interface probed by ET(30) is similar to those of bulk $\mathrm{CCl}_{4}$ and butyl ether, i.e. the same as the DEPNA results reported in this paper. Since DEPNA and ET(30) have different solvatochromism, i.e. DEPNA red shifts and ET(30) blue shifts with increasing solvent polarity, the ET(30) results together with the DEPNA results show that the polarity results are general.

The observation that the polarity of the air/water interface is less than that of bulk water is consistent with expectations. ${ }^{16,37,51}$ However, the finding that the polarity at the air/water interface is comparable to that of bulk nonpolar solvents, i.e. $\mathrm{CCl}_{4}$ and butyl ether, is perhaps surprising at first glance. In the bulk solution the polar solute molecule experiences both local and long-range interactions, whereas at the air/water interface the solute lacks "one-half" of the long-range interactions and experiences a change in its local solvation compared with that in the bulk.

\section{Conclusion}

The polarity of the air/water interface has been determined through the surface-specific measurement of the SH electronic spectrum of the polarity indicator molecule, DEPNA. In terms of the DEPNA $\pi^{*}$ scale, the air/water interface corresponds to a value of $0.22 \pm 0.07$ (373 $\pm 4 \mathrm{~nm})$, similar to very nonpolar solvents, such as carbon tetrachloride $\left(\pi^{*}=0.26,375 \mathrm{~nm}\right)$ and butyl ether $\left(\pi^{*}=0.21,372 \mathrm{~nm}\right)$. The DEPNA results together with the ET(30) results, which will be reported elsewhere, show that the polarity results of the air/water interface are general. This study puts the polarity of the air/water interface on a quantitative polarity scale. Analogous to the value of bulk solvent polarity in describing equilibrium and dynamic processes 
in the bulk, the development of an interface polarity scale permits correlations between various interfacial molecular properties, interfacial reaction equilibrium constants, and reaction rate constants.

Acknowledgments. The authors thank the Division of Chemical Science of the Department of Energy for its support and the National Science Foundation for equipment support. Thanks also go to Dr. Ruo Xu and Shisun (Xuegong) Lei for help in the synthesis of DEPNA.

\section{References and Notes}

(1) Berthelot, M.; De Saint-Gilles, L. P. Ann. Chim. Phys. 3. Sér. 1862, 65,385 .

(2) Berthelot, M.; De Saint-Gilles, L. P. Ann. Chim. Phys. 3. Sér. 1862, 66,5 .

(3) Berthelot, M.; De Saint-Gilles, L. P. Ann. Chim. Phys. 3. Sér. 1863, $68,255$.

(4) Simon, J. D. Acc. Chem. Res. 1988, 21, 128.

(5) Rettig, W.; Baumann, W. In Progress in Photochemistry and Photophysics; Rabek, J. F., Ed.; CRC Press: Boca Raton, FL, 1989; Vol. 6.

(6) Claisen, L. Justus Liebigs Ann. Chem. 1896, 291, 25.

(7) Wislicenus, W. Justus Liebigs Ann. Chem. 1896, 291, 147.

(8) Knorr, L. Justus Liebigs Ann. Chem. 1896, 293, 70.

(9) Recent comprehensive reviews can be found in the special issue of Chem. Rev. 1992, 92 (3).

(10) Reichardt, C. Chem. Rev. 1994, 94, 2319.

(11) Reichardt, C. Solvent and Solvent Effects in Organic Chemistry, 2nd ed.; VCH Publishers: Weinheim, 1988; Chapter 6.

(12) Kamlet, M. J.; Abboud, J. L.; Taft, R. W. In Progress in Physical Organic Chemistry; Cohen, S. G., Streitwieser, A., Taft, R. W., Eds.; Wiley: New York, 1981; Vol. 13, p 485.

(13) Buncel, E.; Rajagopal, S. Acc. Chem. Res. 1990, 23, 226.

(14) Marcus, Y. Chem. Soc. Rev. 1993, 409.

(15) Langan, J. G.; Sitzmann, E. V.; Eisenthal, K. B. Chem. Phys. Lett. 1984, 110, 521

(16) Bayliss, N. S.; McRae, E. G. J. Phys. Chem. 1954, 58, 1002.

(17) Ehrenson, S. J. Am. Chem. Soc. 1981, 103, 6036.

(18) Brady, J. E.; Carr, P. W. J. Phys. Chem. 1985, 89, 5759.

(19) Kamlet, M. J.; Hall, T. N.; Boykin, J.; Taft, R. W. J. Org. Chem. 1979, 44, 2599

(20) Buncel, E.; Rajagopal, S. J. Org. Chem. 1989, 54, 798.

(21) Shen, Y. R. Annu. Rev. Phys. Chem. 1989, 40, 327.

(22) Corn, R. M.; Higgins, D. A. Chem. Rev. 1994, 94, 107.

(23) Richmond, G. L.; Robinson, J. M.; Shannon, V. L. Prog. Surf. Sci. 1988, 28,1 .
(24) Eisenthal, K. B. Acc. Chem. Res. 1993, 26, 636.

(25) Hicks, J. M.; Kemnitz, K.; Eisenthal, K. B.; Heinz, T. F. J. Phys. Chem. 1986, 90, 560 .

(26) Lay, M. B.; Drummond, C. J.; Thistlethwaite, P. J.; Grieser, F. J. Colloid Interface Sci. 1989, 128, 602.

(27) Zachariasse, K. A.; Phuc, N. v.; Kozankiewicz, B. J. Phys. Chem. 1981, 85, 2676.

(28) Drummond, C. J.; Grieser, F.; Healy, T. W. Faraday Discuss. Chem. Soc. 1986, 81, 95 .

(29) Warr, G. G.; Evans, D. F. Langmuir 1988, 4, 217.

(30) Michels, J. J.; Dorsey, J. G. Langmuir 1990, 6, 414.

(31) Lindley, S. M.; Flowers, G. C.; Leffler, J. E. J. Org. Chem. 1985 50,607 .

(32) Perera, J. M.; Stevens, G. W.; Grieser, F. Colloids Surf., A 1995 $95,185$.

(33) Zhao, X.; Subrahmanyan, S.; Eisenthal, K. B. Chem. Phys. Lett. 1990, 171, 558 .

(34) Zhao, X. L.; Ong, S. W.; Eisenthal, K. B. Chem. Phys. Lett. 1993, 202, 513.

(35) Wang, H.; Zhao, X. L.; Eisenthal, K. B. To be published.

(36) Xiao, X.-D.; Vogel, V.; Shen, Y. R. Chem. Phys. Lett. 1989, 163 , 555.

(37) Shi, X.; Borguet, E.; Tarnovsky, A. N.; Eisenthal, K. B. Chem. Phys. 1996, 205, 167.

(38) Bloembergen, N. Nonlinear Optics; Benjamin: New York, 1965.

(39) Heinz, T. F. Second-Order Nonlinear Optical Effects at Surfaces and Interfaces; Elsevier: Amsterdam, 1991.

(40) Shen, Y. R. Principles of Nonlinear Oprics; Wiley: New York, 1984.

(41) Marowsky, G.; Gierulski, A.; Dick, B. Optics Commun. 1985, 52, 339.

(42) Tom, H. W. K.; Heinz, T. F.; Shen, Y. R. Laser Chem. 1983, 3 ,

(43) Petralli, T.; Wong, T. M.; Byers, J. D.; Yee, H. I.; Hicks, J. M. J. Phys. Chem. 1993, 97, 1383

(44) Nguyen, D. C.; Muenchausen, R. E.; Keller, R. A.; Nogar, N. S. Opt. Commun. 1986, 60, 111.

(45) Yam, R.; Berkovic, G. Langmuir 1993, 9, 2109.

(46) Behr, L. C.; Kirry, J. E.; MacDonald, R. N.; Todd, C. W. J. Am. Chem. Soc. 1946, 68, 1296.

(47) Kamlet, M. J.; Taft, R. W. J. Am. Chem. Soc. 1976, 98, 377.

(48) Adamson, A. W. Physical Chemistry of Surfaces, 4th ed.; John Wiley \& Sons: New York, 1982; Chapter 3, p 73.

(49) Bhattacharyya, K.; Sitzmann, E. V.; Eisenthal, K. B. J. Chem. Phys. 1987, 87, 1442

(50) Essfar, M.; Guiheneuf, G.; Abboud, J. M. J. Am. Chem. Soc. 1982 104, 6786

(51) Wilson, M. A.; Pohorille, A. J. Chem. Phys. 1991, 95, 6005. 